\section{A. Hasman}

Dept. of Medical Informatics, University of Limburg, Maastricht, The Netherlands

\section{Introduction}

The development of methods for the processing of images and signals is one of the oldest research topics in medical informatics. Already early after the introduction of computers, methods were either developed for the analysis of, for example, signals generated by laboratory equipment, and of ECGs and EEGs, adopted from other disciplines. In parallel, image processing methods were developed, e.g., for analyzing cells, chromosomes and images from cytology, radiology and nuclear medicine.

The goals of signal and image analysis varied, but classification of signal and image components was an important topic. Both methods that were developed with specific applications in mind and generic methods have to be validated and evaluated. Algorithms usually compute a number of parameter values that are to be determined from available signals. When the algorithm is very elaborate, the number of parameters may become so large that the determination of their optimal values becomes impractical; the time to perform the analysis with sets of values for each of the various parameters becomes too long. Therefore, many complex algorithms usually use a number of parameters (thresholds) whose values have been determined by trial and error.

It was realized early on that, in order to evaluate the performance of the algorithms, data sets should be used other than those used for training the algorithms. Therefore, a distinction

\title{
Synopsis
}

\section{Image and Signal Processing}

between training set and test set was made. Unfortunately, this distinction is not always apparent from publications. Because of the large number of thresholds in the algorithm, and the different circumstances in which the data have been obtained, it is difficult to assess whether the size of the test set is large enough to assess the performance of the algorithm. The sizes of the data sets discussed in the contributions of this section ranged from 27 to 1000 .

Fully automated analysis of signals and images has always been one of the aims of research projects. In signal analysis, fully automated procedures were shown to be possible: ECGs, for instance, can be automatically analyzed and classified without human intervention. In image analysis, however, better results were obtained with interactive systems; optimal use was made of the combined capabilities of man and machine. For a large number of applications, however, it is rather time consuming when performed in an interactive way. In these situations, solutions are needed in which the human involvement is kept to a minimum.

Knowledge about the application domain is often incorporated in the algorithms. Increasingly, knowledge is explicitly represented in image and signal processing, e.g., to determine where in the image or the signal a certain procedure has to be applied. It has been shown in AI that relatively simple control strategies can lead to complex behavior. In addition, proper knowledge representation is important for solving an image understand- ing problem. Models (e.g., in the form of semantic nets) are used to represent domain knowledge. Recognition is then a matter of graph matching. Most of the mentioned issues are present in the contributions in this section.

The contributions can be divided into two groups:

- The first group consists of approaches which provide output that is subsequently used by the user, or by a program, to perform related tasks. One example is the threedimensional visualization of the mandible to support clinical management in dentistry [1]. The other contribution concerns the determination of the ribcage boundary [2]. The ribcage boundary provides useful information on the location, shape and size of the lung fields. This information is required for the successful performance of computer-aided programs for detecting abnormalities in chest images.

- Quantification is the topic of the second group. This group consists of three contributions. The first one is concerned with the automatic determination of heart chamber volumes and ejection fractions [3]. These quantities can be obtained when a method is available that automatically detects the endocardial and epicardial borders of the left ventricle. The aim of the second contribution is to quantify interstitial chronic renal damage [4]. There is a relationship between chronic renal damage and renal function, and the quality of the method was expressed in terms of the extent to 
which the isotopic glomerular filtration rate could be predicted. The third contribution describes an attempt to estimate the cardiac output continuously using the arterial pressure waveform [5]. In this case, shape analysis was performed of the arterial pressure waveform, the idea being that each of the resulting typical shapes predicts a certain cardiac output.

- The last contribution concerns the automated extraction, labelling and analysis of the coronary vasculature fromarteriograms [6]. This contribution contains aspects of both groups: it offers support to users for performing other tasks, but it also quantifies.

\section{Three-dimensional Visualization of the Mandible}

The authors [1] assume that the basis of appropriate clinical management in dental practices is a continuous holistic understanding of a patient's clinical situation. All relevant information should be provided in one view'. The goal of the research work was to offer a tool for the visualization of all relevant periodontal findings in a three-dimensional form.

The program presents the individual pathology of a patient using a reference jaw model. This reference model was constructed from the slices obtained from an artificial skull. These slices were photographed and scanned, thus giving rise to a large set of volume data. These data were converted into a surface representation that can subsequently be processed by common shading tools. To reconstruct the teeth, an algorithm was developed that determines the tooth contour automatically. The contours of the mandible bone and the gingiva were traced manually. With the help of this model periodontal pathologies can be simulated. An illustration is presented of facial bone pockets/vertical bone-loss visualization. Relevant control points of the patch mesh used for the surface representation are moved to new positions in such a way that the bone deformation becomes clearly visible.

The three-dimensional display contributes, according to the authors, to an improved perception of the clinical situation. Images can, for example, be constructed showing the progression of periodontal disease. These images can be shown in a movie loop, or can be superimposed to indicate changes. The authors have not yet investigated whether the visualization indeed improves patient care.

The system can also be used for educational purposes, for example as a component of interactive teachware to visualize the simulated results of students' treatment proposals.

\section{Determination of the Ribcage Boundary}

Computer-aided diagnosis programs for detecting abnormalities in chest images require knowledge about the location, shape and size of lung fields. When such programs are provided with knowledge about the ribcage boundary, they can function more reliably.

Several techniques for the determination of the ribcage boundary have been proposed, ranging from simple thresholding procedures to edge-detection algorithms based on minima in second derivatives. In this contribution a set of 1000 chest radiographs was used [2]. It exemplifies the problems with multi-parameter algorithms as mentioned earlier. The first problem to be attacked is the determination of the lung top. A combination of horizontal and vertical profiles is used. With the help of a vertical profile, possible candidate positions for the lung top are obtained. The horizontal profiles are used to discriminate between candidates actually located in the neck area, the lung top, or the lung area. The neck area appears to have both a high standard deviation and a high maximum difference of pixel values along the horizontal profile. Whether the candidate is situated in the lung area could be determined by correlating the corresponding profile with a typical lung profile. This latter profile was obtained from a position in the chest image that certainly was situated within the lung area. Criterion functions with which it is possible to establish whether the candidate under consideration is indeed the lung top, were determined using 120 chest radiographs. It was verified using (another?) set of 120 cases whether the actual position of the lung top was close to the measured position.

After the determination of a few other landmarks, the edges are detected using the profile obtained from a suitably oriented rectangular region of interest. The minima in the second derivative of the profile are candidates of edge locations. Another criterion function is used to select the correct minimum. Finally, a fourth-degree polynomial is used to produce a smooth ribcage boundary.

The accuracy of the resulting boundaries was judged on a five-point scale by three radiologists and two physicists using the 1000 images. The overall accuracy of the method appeared to be $96 \%$. The authors suggest applying the method to routine chest images in a real clinical environment to determine the performance under normal clinical conditions.

\section{Automatic Volume Computation of the Left Ventricle}

This contribution considers the fully automatic detection of the endocardial and epicardial borders of the left ven- 
tricle from images obtained with ultrafast computed tomography [3]. The usual method of assessing these images is by the use of manual tracing methods. These methods are time-consuming and subject to individual variability. The method presented in this contribution is fully automatic. First, the position of the heart is determined, then the epicardial and endocardial contours.

In determining the location of the heart, the chest wall has to be located first. A model of the chest wall was built by manually tracing the chest wall in a training set of images of the basal level of four randomly chosen subjects and then averaging them to produce a normalized model. This model was subsequently used to determine the position of the chest wall in other images.

The position and extent of the left lung was determined using a suitably chosen seedpoint and by applying region growing. A similar method was used for determining the epicardial surface. A rectangular region was defined around the estimated epicardial contour. Accurate myocardial contours were then determined within this region of interest. Based on the earlier found epicardial contour, seedpoints were determined in the left and right ventricle. Again, region growing was used to identify the borders. With the help of a graph-searching procedure optimal estimates of the true contours were obtained. These contours were then used as models for the next image slice to be analyzed.

The contours are displayed superimposed on the original image. The confidence in the correctness of the contour was also calculated and displayed. In this way, the operator is provided with information concerning those portions of the contour where modifications may be required.

The method was tested by comparing the cross-sectional areas and the chamber volumes computed with this technique, with those from manually traced images using the images of 27 patients. It was concluded that the algorithm provides an accurate estimation of areas and volumes. It is suggested that the technique can also be used in other imaging modalities for contour identification.

\section{Quantification of Interstitial Chronic Renal Damage}

The goal of the study was to investigate whether chronic renal damage could be automatically measured by means of texture analysis [4]. Use was made of granulometry based on the principles of mathematical morphology as introduced by Matheson.

The study was performed using 35 biopsies that were diagnosed as chronic evolutive nephropathy. The biopsies were sectioned and stained. In each patient the glomerular filtration rate (GFR) was determined at the time of biopsy. Images were digitized at $256 \times 256$ pixels. The negative of the digitized image was stored and analyzed.

The image can be regarded as a three-dimensional volume, with the surface being determined by the greylevel intensity. In granulometry, geometric objects are used to study an image. In this case the object is a disc with a certain diameter. At each pixel position, this horizontal disc is moved in the vertical direction until it hits the boundary of the three-dimensional volume. In this way the volume is divided into two parts: the volume above the discs and the volume below it. By increasing the radius of the disc, the volume below the discs gradually decreases. A spectrum is defined indicating the decrease in volume as a function of the radius of the disc. In this study the range of the radius was varied from 1 to 60 . In this way 60 variables were obtained with an assigned value equal to the volume re- duction they produce.

The variables were used as independent variables in a regression analysis with the measured GFR as the dependent variable. Five variables appeared to predict the GFR.

When comparing these results with an existing manual method it appeared that the granulometric method estimated the GFR more accurately than the manual method. Based on this finding it is concluded that a fully automatic method was designed that precisely quantifies interstitial chronic renal damage.

\section{Determination of Continuous Cardiac Output}

Continuous determination of cardiac output is important for monitoring acutely ill patients. The cardiac output has been estimated by several authors from the arterial pressure waveform using a simple model for the arterial system. These methods are not suitable for critically ill patients in whom the hemodynamic state is variable.

In this contribution, pattern recognition techniques are used to derive the cardiac output from the arterial pressure waveform [5]. The idea is that a limited number of typical waveforms can be identified by which new waveforms can be classified.

Each waveform from the training set is represented by a number of features. Some kind of singular value decomposition is applied to obtain a set of what are called typical shapes. New pressure waveforms are assigned to that typical shape to which it is closest. This closest typical shape defines the cardiac output of the new pressure waveform. The approach is similar to a study performed by Muijtjens et al. with respect to following markers for estimating deformation $[7,8]$.

A training set of 100 'patients' was 
used for the singular value decomposition to obtain the typical shapes. The method was evaluated using a different set of 420 simulated patients, using a comprehensive pulsatile-flow cardiovascular model. It was concluded that the presented method was more accurate than three methods described in the literature.

The method was further evaluated in an animal study. Cardiac output was measured with an ultrasonic flow probe. The size of each class belonging to a typical shape was taken such that the corresponding cardiac outputs within the class did not vary by more than $5 \%$. It was determined that the overall accuracy of the procedure was within the desired value of $25 \%$.

In order to obtain enough typical shapes a large number of patients is required. It was estimated that at least 400 patients are necessary for obtaining a complete set of waveforms.

\section{Automated Extraction, Labelling and Analysis of the Coronary Vasculature}

In this contribution techniques are described that can extract, label and analyze the coronary vasculature from arteriograms in an automated, quantitative way [6]. The first part is concerned with the extraction of the coronary skeleton.

The procedure starts with a useridentified seedpoint, a user-defined tracking direction and a circular template with a diameter that is slightly larger than the local diameter of the vessel segment. The vessel boundary is detected by applying a first derivative function of the grey value profile along the circumference of the template. A skeleton point is defined as the midpoint of the boundary points. The template is repositioned to the newly found skeleton point and the radius is adapted.

The tracking of a vessel segment is terminated, among others, when the template size becomes smaller than a preset threshold value, a value determined from 100 arteriograms. After the complete skeleton is extracted, the precise vessel boundaries are detected using information about the first and second derivative of the grey-value profiles. The labelling procedure then follows. Use is made of a priori knowledge about the coronary anatomy. A graph is constructed of the relations between vessel segments in the skeleton. A similarly labelled graph is available for the model of the coronary anatomy. These two graphs are matched using a dissimilarity measure. Since a large number of matches is feasible, the dissimilarity measure was used to exclude a large number of candidate matches. The remaining candidates were evaluated using the $\mathrm{A}^{*}$-algorithm.

The accuracy of the procedure in determining local vessel diameters was evaluated. A systematic bias in the measurements was apparent. About $85 \%$ of the visible segments were tracked. After manual deletion of false background structures, $86 \%$ of the LAD was correctly labelled. It is concluded that it is possible to automatically assess quantitatively vessel diameters and stenoses from the entire coronary tree.

\section{Conclusion}

A number of contributions have been discussed that were rather application-oriented. The methods used in the various contributions show that image and signal processing applications can be advantageous in routine clinical practice. As was stated earlier, the final performance will only be clear after being applied to large collections of cases. In addition, the influence should be assessed of these kinds of applications on the quality of clinical care. Are the benefits of introduc- ing these systems in practice large enough? How does the organization accept the new application? With these contributions at least an important step has been set in the right direction.

\section{References}

1. Seipel S, Wagner I-V, Koch S, Schneider W. Three-dimensional visualization of the mandible: a new method for presenting the periodontal status and diseases. Comput Methods Programs Biomed 1995;4:51-7.

2. Xin-Wei Xu, Kunio Dói. Image feature analysis for computer-aided diagnosis: accurate determination of ribcage boundary in chest radiographs. Med Phys 1995;22:617-26.

3. Dove EL, Philip K, Gotteiner NL, Vonesh MJ, Riembergen JA, Reed JE, Stanford W, McPherson DD, Chandran B. A method for automatic edge detection and volume computation of the left ventricle from ultrafast computed tomagraphic images. Invest Radiol 1994;1 1:945-54.

4. Moreso F, Serón D, Vitriá J, Grinyó JM, Colomé-Serra FM, Parés N, Serra J. Quantification of interstitial chronic renal damage by means of texture analysis. Kidney Int 1994;46:1721-7.

5. Martin JF, Volfson LB, Kirzon-Zolin VV, Schukin VG. Application of pattern recognition and image classification techniques to determine continuous cardiacoutput from the arterial pressure waveform. IEEE Trans Biomed Eng 1994;41:913-20.

6. Dumay ACM, Gerbrands JJ, Reiber JHC. Automated extraction, labelling and analysis of the coronary vasculature from arteriograms. Int J Card Imaging 1994;10:205-15.

7. Muijtjens $A M M$, Roos JM, Prinzen TT, Hasman A, Reneman RS, Arts T. Noise reduction in estimating cardiac deformation from marker tracks. Am J Physiol 1990;258:H599-605.

8. Muijtjens AAM, Roos JMA, Arts T, Hasman A. Extrapolation of incomplete marker tracks by lower rank approximation. Int J Biomed Comput 1993;33:21939.

Address of the author:

A. Hasman,

Dept. of Medical Informatics,

University of Limburg,

P.O. Box 616,

6200 MD Maastricht,

The Netherlands. 